\title{
Ghrelin Attenuates Brain Injury after Traumatic Brain Injury and Uncontrolled Hemorrhagic Shock in Rats
}

\author{
Lei Qi, ${ }^{1,2,3}$ Xiaoxuan Cui, ${ }^{1,2}$ Weifeng Dong, ${ }^{1,2}$ Rafael Barrera, ${ }^{2}$ Jeffrey Nicastro, ${ }^{2}$ Gene F Coppa, ${ }^{2}$ Ping Wang, ${ }^{1,2}$ \\ and Rongqian $W u^{1,2}$
}

${ }^{1}$ The Feinstein Institute for Medical Research, Manhasset, New York, United States of America; ${ }^{2}$ Department of Surgery, North Shore University Hospital and Long Island Jewish Medical Center, Manhasset, New York, United States of America; ${ }^{3}$ Department of Neurosurgery, First Affiliated Hospital of Medical School, Xi'an Jiaotong University, Xi'an, Shaanxi, China

\begin{abstract}
Traumatic brain injury (TBI) and hemorrhagic shock often occur concomitantly due to multiple injuries. Gastrointestinal dysfunction occurs frequently in patients with TBI. However, whether alterations in the gastrointestinal system are involved in modulating neuronal damage and recovery after TBI is largely neglected. Ghrelin is a "gut-brain" hormone with multiple functions including antiinflammation and antiapoptosis. The purpose of this study was to determine whether ghrelin attenuates brain injury in a rat model of TBI and uncontrolled hemorrhage (UH). To study this, brain injury was induced by dropping a 450-g weight from $1.5 \mathrm{~m}$ onto a steel helmet attached to the skull of male adult rats. Immediately after TBI, a midline laparotomy was performed and both lumbar veins were isolated and severed at the junction with the vena cava. At $45 \mathrm{~min}$ after TBI/UH, ghrelin (4, 8 or 16 nmol/rat) or $1 \mathrm{~mL}$ normal saline (vehicle) was intravenously administered. Brain levels of TNF- $\alpha$ and IL-6, and cleaved PARP- 1 levels in the cortex were measured at $4 \mathrm{~h}$ after TBI/UH. Beam balance test, forelimb placing test and hindlimb placing test were used to assess sensorimotor and reflex function. In additional groups of animals, ghrelin ( $16 \mathrm{nmol} / \mathrm{rat}$ ) or vehicle was subcutaneously (s.c.) administered daily for $10 \mathrm{~d}$ after TBI/UH. The animals were monitored for $28 \mathrm{~d}$ to record body weight changes, neurological severity scale and survival. Our results showed that ghrelin downregulated brain levels of TNF- $\alpha$ and IL-6, reduced cortical levels of cleaved PARP-1, improved sensorimotor and reflex functions, and decreased mortality after TBI/UH. Thus, ghrelin has a great potential to be further developed as an effective resuscitation approach for the trauma victims with brain injury and severe blood loss.
\end{abstract}

Online address: http://www.molmed.org

doi: 10.2119/molmed.2011.00390

Traumatic brain injury (TBI) remains a major public health problem globally (1-5). The Centers for Disease Control and Prevention (CDC) has estimated that each year, approximately 1.5 million Americans survive a TBI, among whom approximately 230,000 are hospitalized. Approximately 53,000 Americans die each year following traumatic brain injury, representing one third of all injuryrelated deaths (3). Moreover, TBI resulting from either blast injury or vehicle accidents represents important issues for the combat medic. This has become in- creasingly important with the escalation of the use of improvised explosive devices (IEDs) by insurgents and terrorists against military and civilian personnel. As a matter of fact, TBI has been called the "signature wound" of the Iraqi and Afghanistan conflicts. However, no drugs are currently approved for the treatment of TBI. At present, the standard of care for TBI patients is simply supportive care, monitoring of blood pressure, monitoring of blood oxygen levels, and, in some cases, monitoring intracranial pressure (ICP). Thus, there is

Address correspondence to Rongqian Wu, Laboratory of Surgical Research, The Feinstein Institute for Medical Research, 350 Community Drive, Manhasset, NY 11030 , USA. Phone: (516) 562-2390; Fax: (516) 562-2396; E-mail: rwu@nshs.edu.

Submitted October 10, 201 1; Accepted for publication November 29, 2011 ; Epub (www.molmed.org) ahead of print December 6, 2011.

The Feinstein Institute North for Medical Research North Shore

an urgent unmet medical need for specific and innovative therapies for TBI.

TBI and hemorrhagic shock, the most common causes of trauma deaths, often occur concomitantly due to multiple injuries $(6,7)$. Hemorrhagic shock markedly exacerbates secondary damage in the traumatically injured brain and doubles TBI mortality $(6,8)$. TBI, on the other hand, impairs shock compensation. In this regard, a therapeutic intervention to treat posttraumatic hypotension and prevent secondary ischemia would be a powerful tool to improve outcome after brain injury.

Ghrelin, a novel "gut-brain" hormone, was first identified in 1999 as an endogenous ligand for the growth hormone secretagogue receptor type 1a (GHSR-1a, that is, ghrelin receptor) (9). It was originally reported to induce growth hormone $(\mathrm{GH})$ release through pituitary GHSR-1a stimulation (10-12). However, a large body of evidence has indicated other physiological 
functions of ghrelin mediated by the central and peripheral ghrelin receptors $(13,14)$. Our recent studies have shown that ghrelin ameliorates gut ischemia reperfusion or sepsis-induced organ injury and mortality via stimulation of ghrelin receptors in the brain (15-18). Ghrelin has been shown to inhibit apoptosis in neuronal cells during oxygen-glucose deprivation (19) and protect cortical neuron against focal ischemia/reperfusion (I/R) in rats $(20,21)$. Using a mouse model of TBI, Bansal et al. has shown that ghrelin prevents TBI-induced intestinal dysfunction in mice (22). However, whether ghrelin attenuates brain injury following TBI and hemorrhagic shock remained unknown. The purpose of this study was to evaluate the efficacy of ghrelin using a highly military relevant experimental model of TBI combined with uncontrolled hemorrhagic shock in rats.

\section{MATERIALS AND METHODS}

\section{Experimental Animals}

Adult male Sprague-Dawley rats, purchased from Charles River Labs. (Wilmington, MA, USA), were used in this study. The rats were housed in a temperature-controlled room on a 12-h light/dark cycle and fed on a standard Purina rat chow diet. The rats were fasted for $12 \mathrm{~h}$ before the procedure. Because rats are social animals, they were group housed in this study. Animal experimentation was carried out in accordance with the Guide for the Care and Use of Laboratory Animals (Institute of Laboratory Animal Resources). This project was approved by the Institutional Animal Care and Use Committee (IACUC) of The Feinstein Institute for Medical Research.

\section{Rat Model of TBI and Uncontrolled Hemorrhagic Shock}

Male Sprague-Dawley rats weighing 325-375 g were anesthetized with isoflurane inhalation. Catheters (PE-50) were placed in a femoral vein. TBI was induced by Marmarou's model (23).

Briefly, a midline scalp incision was performed followed by periosteal elevation to expose the central area of the skull vault between the coronal and lambdoid sutures. A helmet (that is, a stainless steel disc $10 \mathrm{~mm}$ in diameter and $3 \mathrm{~mm}$ thick) was firmly fixed by dental acrylic to this central portion of the skull vault. A 450-g weight was allowed to drop freely from $1.5 \mathrm{~m}$ onto the steel helmet attached to the skull of the rats. This model induces diffuse cellular and axonal injury in forebrain structures such as sensorimotor cortex and hippocampus but limited brainstem damage $(23,24)$. Immediately after TBI, the rat was subjected to nonlethal uncontrolled hemorrhage (UH) induced by venous injury as we described recently (25). Briefly, a midline laparotomy was performed and both lumbar veins were isolated and severed at the junction with the vena cava. The abdomen was kept open but covered with a saline wet gauze for $20 \mathrm{~min}$, and it was closed in layers thereafter. At $45 \mathrm{~min}$ after TBI and UH, the animals were intravenously resuscitated with $1 \mathrm{~mL}$ normal saline (that is, low volume resuscitation) with or without various doses of ghrelin (4, 8 and $16 \mathrm{nmol} /$ rat, Phoenix Pharmaceuticals, Belmont, CA, USA) via the femoral venous catheter over $10 \mathrm{~min}$. The doses of ghrelin used in this study were chosen based on our previous experience in sepsis and ischemia reperfusion injury $(15,26,27)$. Sham animals (that is, control animals) were anesthetized with isoflurane inhalation and underwent the same surgical procedure with the exception of TBI and hemorrhage. At $4 \mathrm{~h}$ after resuscitation, the laparotomy incision was reopened and free intraperitoneal blood collected on preweighed pieces of gauze. The amount of blood loss was determined by the difference in wet and dry weights. The total bleedout volume in this model was $7.0 \pm 0.1 \mathrm{~mL} / \mathrm{rat}$. Owing to the use of the helmet, most of the animals did not have grossly visible damage to the skull. But mild brain swelling was visible at $4 \mathrm{~h}$ after TBI and UH.

\section{Determination of Brain Levels of TNF- $\alpha$ and IL-6}

At $4 \mathrm{~h}$ after TBI $+\mathrm{UH}$ or sham operation, the brain was rapidly harvested.

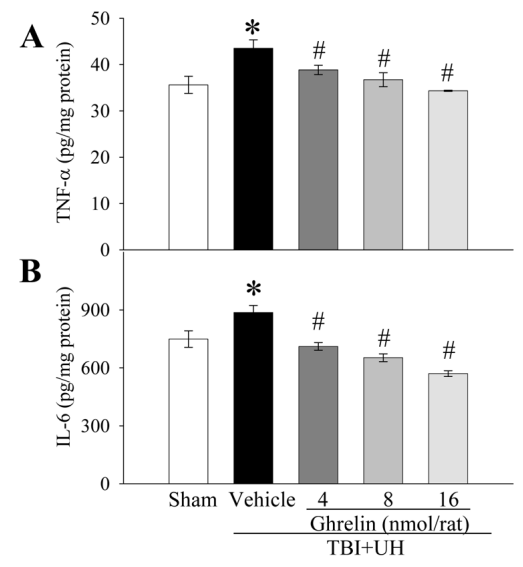

Figure 1. Downregulation of brain inflammation by ghrelin after TBI and UH. Alterations in brain levels of TNF- $\alpha$ (A) and IL-6 (B) in sham-operated animals (Sham) and $\mathrm{TBI}+\mathrm{UH}$ animals treated with normal saline (Vehicle) or ghrelin (Ghrelin) at $4 \mathrm{~h}$ after $\mathrm{TBI}+\mathrm{UH}$. Data are presented as means \pm SE $(n=5-6)$ and compared by one-way ANOVA and Student-Newman-Keuls test: ${ }^{*} P<0.05$ versus Sham group; ${ }^{*} P 0.05$ versus Vehicle group.

Brain samples were longitudinally cut along the middle line. The left hemisphere was excised, rinsed of blood, homogenized with polytron in a homogenization buffer (phosphate-buffered saline solution, containing $0.05 \%$ Triton $\mathrm{X}-100$ and a protease inhibitor cocktail; $\mathrm{pH}, 7.2 ; 4^{\circ} \mathrm{C}$ ), and sonicated for $10 \mathrm{~s}$. Homogenates were centrifuged at $16,000 \mathrm{~g}$ for $20 \mathrm{~min}$, and TNF- $\alpha$ and IL-6 levels were quantified using an enzyme-linked immunosorbent assay (ELISA) kit specifically for rat TNF$\alpha$ or IL-6 (BD Biosciences, San Diego, CA, USA). The assay was carried out according to the instructions provided by the manufacturer. Brain levels of TNF- $\alpha$ and IL-6 were normalized to the protein concentration in the sample.

\section{Western Blotting Analysis of Cleaved Poly (ADP-ribose) Polymerase- 1 (PARP-1)}

At $4 \mathrm{~h}$ after TBI $+\mathrm{UH}$ or sham operation, the cortex was harvested. The samples were lysed and homogenized in lysis buffer (10 mmol/L TBS, $1 \mathrm{mmol} / \mathrm{L}$ EDTA, $1 \mathrm{mmol} / \mathrm{L}$ EGTA, $2 \mathrm{mmol} / \mathrm{L} \mathrm{Na}$ 
Orthovanadate, $0.2 \mathrm{mmol} / \mathrm{L}$ PMSF, $2 \mu \mathrm{g} / \mathrm{mL}$ leupeptin, $2 \mu \mathrm{g} / \mathrm{mL}$ aprotinin, $1 \%$ Triton-X 100) for $30 \mathrm{~min}$ on ice, and cleared by centrifugation at $16,000 \mathrm{~g}$ for $20 \mathrm{~min}$ at $4^{\circ} \mathrm{C}$. Homogenates containing $50 \mu \mathrm{g}$ protein were fractionated on $4-12 \%$ Bis-Tris gel and transferred to $0.45 \mu \mathrm{m}$ nitrocellulose membrane. Nitrocellulose blots were blocked by incubation in TBST (10 mmol/L Tris-HCl, pH 7.5, 150 mmol/L $\mathrm{NaCl}, 0.1 \%$ Tween 20 ) containing 5\% milk for $1 \mathrm{~h}$. Blots were incubated with rabbit anti-cleaved PARP (Asp214) antibody (1:300, Cell Signaling Technology Inc., Danvers, MA, USA) overnight at $4^{\circ} \mathrm{C}$. The blots were then washed in TBST 3 times for $10 \mathrm{~min}$. Blots were incubated with HRP-labeled goat anti-rabbit IgG for $1 \mathrm{~h}$ at room temperature, and then washed 3 times in TBST for $10 \mathrm{~min}$. A chemiluminescent peroxidase substrate (ECL plus, Amersham Biosciences, Piscataway, NJ) was applied according to the manufacturer's instructions, and the membranes were exposed briefly to X-ray film. The band densities were normalized by $\beta$-actin using the Bio-Rad image system.

\section{Morphological Examination}

The morphological alterations in the brain were examined at $4 \mathrm{~h}$ after TBI and UH. Brain samples were prepared by transcardial perfusion immediately with cold normal saline followed by $4 \%$ paraformaldehyde (Sigma, St. Louis, MO, USA) in $0.1 \mathrm{~mol} / \mathrm{L}$ sodium phosphate buffer ( $\mathrm{pH}$ 7.4). After removing from the cranial cavity, the brains were postfixed in $4 \%$ paraformaldehyde at room temperature overnight and were infiltrated in 25\% sucrose in $0.1 \mathrm{~mol} / \mathrm{L} \mathrm{PBS}$ at $4^{\circ} \mathrm{C}$ for $48 \mathrm{~h}$ thereafter. The brain samples were embedded in paraffin and coronal sections at the level $-4 \mathrm{~mm}$ from the bregma were collected. The tissue blocks were then sectioned at a thickness of $5 \mu \mathrm{m}$, floated on warm water, and transferred to glass slides, where they were stained with hematoxylin and eosin (H-E), dehydrated, and covered with coverslips. Morphological examinations were performed using a light microscope and then documented with photographs in a blinded manner.

\section{Determination of Brain $\beta$-Amyloid Precursor Protein ( $\beta$-APP) Levels by Immunohistochemistry}

The brain sections as collected above were dewaxed and rehydrated, followed by microwave antigen retrieval procedure. Endogenous peroxidase and nonspecific binding sites were blocked using $2 \% \mathrm{H}_{2} \mathrm{O}_{2}$ in $60 \%$ methanol and $3 \%$ normal goat serum, respectively. The sections were then incubated with rabbit anti- $\beta$-APP polyclonal IgG (1:200, Santa Cruz Biotechnology Inc., Santa Cruz, CA, USA) overnight at $4^{\circ} \mathrm{C}$. After reacting sections with biotinylated anti-rabbit IgG, Vectastain ABC reagent and DAB kit were used to reveal the immunohistochemical reaction (Vector Labs, Burlingame, CA, USA). Normal rabbit IgG was used as primary antibody for negative control. The examination was performed in a blinded manner.

\section{Neurological Severity Scale (NSS)}

Neurological functional impairments and improvements were measured for up to $28 \mathrm{~d}$ after TBI and UH with or without ghrelin treatment by scales of beam balance test, forelimb placing test and hindlimb placing test, indicating sensorimotor functions and reflex. Briefly, the beam balance test examines vestibulomotor reflex activity as the animal balances on a narrow beam $(30 \mathrm{~cm} \times$ $1.3 \mathrm{~cm}$ ) for $60 \mathrm{~s}$. Ability to balance on the beam is scored as follows: animal balances with all four paws on the top of beam, 1; animal puts paws on side of beam or wavers on beam, 2; one or two limbs slip off beam, 3; three limbs slip off beam, 4; animal attempts to balance with paws on beam but falls off, 5; animal drapes over beam, then falls off, 6; animal falls off beam without an attempt to balance, 7. Animals received three training trials before surgery; the score of the last of these was taken as the baseline score. The forelimb placing test is comprised of three subtests. Separate scores are obtained for each forelimb. For the visual placing subtest, the animal is held upright by the examiner and brought close to a table top. Normal placing of

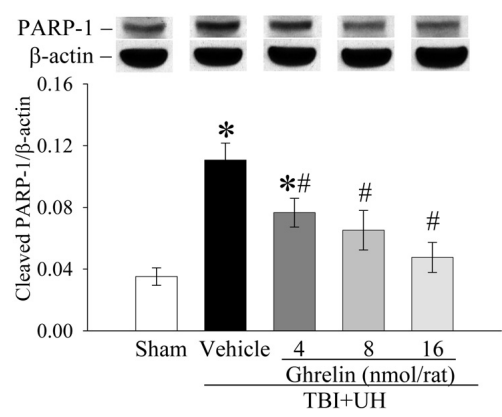

Figure 2. Inhibition of cortical apoptosis by ghrelin after TBI and UH. Alterations in the levels of cleaved PARP1 in the cortex in sham-operated animals (Sham) and TBI+ UH animals treated with normal saline (Vehicle) or ghrelin (Ghrelin) at $4 \mathrm{~h}$ after TBI + $\mathrm{UH}$. Data are presented as means $\pm \mathrm{SE}$ $(n=5-6)$ and compared by one-way ANOVA and Student-Newman-Keuls test: ${ }^{*} P<0.05$ versus Sham group; ${ }^{*} P<0.05$ versus Vehicle group.

the limb on the table is scored as 0 , delayed placing $(<2 \mathrm{~s})$ is scored as 1 , and no or very delayed placing $(>2 s)$ is scored as 2. Separate scores are obtained first as the animal is brought forward and then again as the animal is brought sideways to the table (maximum score per limb, 4; in each case, higher numbers denote greater deficits). For the tactile placing subtest, the animal is held so that it cannot see or touch the table top with its whiskers. The dorsal forepaw is touched lightly to the table top as the animal is first brought forward and then brought sideways to the table. Placing each time is scored as above (maximum score per limb, 4). For the proprioceptive placing subtest, the animal is brought forward only, and greater pressure is applied to the dorsal forepaw; placing is scored as above (maximum score per limb, 2). These subscores are added to give the total forelimb placing score per limb (range, 0-10). The hindlimb placing test is conducted in the same manner as above for the hindlimbs, but involves only tactile and proprioceptive subtests (maximal scores 4 and 2, respectively; total score range, 0-6). The examination was performed in a blinded manner. 


\section{Survival Study}

In additional groups of animals, ghrelin $(16 \mathrm{nmol} / \mathrm{rat})$ or vehicle was subcutaneously (s.c.) administered daily for $10 \mathrm{~d}$ after TBI and UH. The animals were allowed food and water ad libitum and were monitored for $28 \mathrm{~d}$ to record body weight changes, NSS and survival.

\section{Statistical Analysis}

All data are expressed as mean \pm standard error (SE) and compared by oneway ANOVA and the Student-NewmanKeuls test or Student $t$ test. The survival rate was estimated by Kaplan-Meier method and compared by the log-rank test. Differences in values were considered significant if $P<0.05$.

\section{RESULTS}

\section{Effects of Ghrelin on Brain Levels of Inflammatory Cytokines after Traumatic Brain Injury and Uncontrolled Hemorrhagic Shock}

TBI initiates a cascade of inflammatory processes that can serve to exacerbate the initial injury (28). As shown in Figure 1A, brain levels of TNF- $\alpha$ increased by $22 \%$ at $4 \mathrm{~h}$ after TBI and UH $(P<0.05)$. All three doses of ghrelin treatment decreased brain levels of TNF- $\alpha$ after TBI and UH $(P<0.05$, Figure 1A). Similarly, brain levels of IL- 6 increased by $18 \%$ at $4 \mathrm{~h}$ after TBI and UH $(P<0.05)$. All three doses of ghrelin treatment also decreased brain levels of IL-6 after TBI and UH $(P<0.05$, Figure 1B).

\section{Effects of Ghrelin on Cortical Apoptosis after Traumatic Brain Injury and Uncontrolled Hemorrhagic Shock}

Apoptosis is thought to play an important role in both acute and chronic brain injury (29). As shown in Figure 2, cortical levels of cleaved PPAR-1, an indicator of apoptosis (30), increased by more than two-fold at $4 \mathrm{~h}$ after TBI and UH as compared with those in sham-operated animals $(P<0.05)$. Administration of 4,8 and $16 \mathrm{nmol} /$ rat ghrelin reduced cortical apoptosis at $4 \mathrm{~h}$ after TBI and UH by $31 \%, 41 \%$ and $57 \%$, respectively $(P<$ 0.05 , Figure 2).
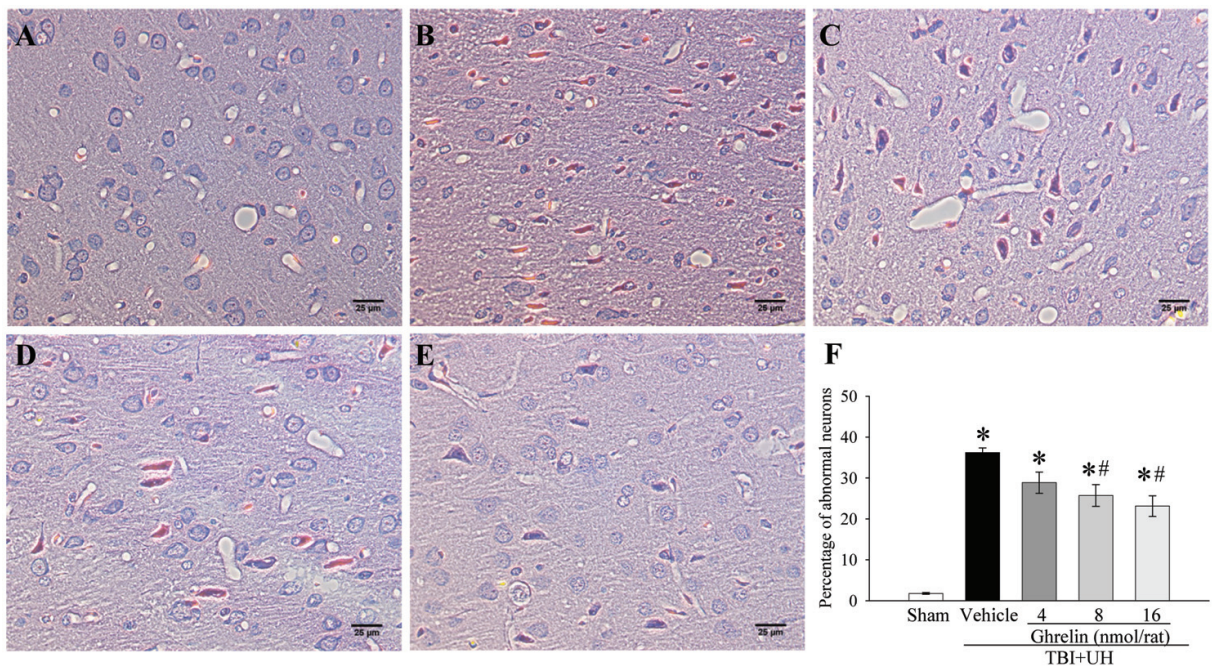

Figure 3. Effects of ghrelin on morphologic photomicrography of the cortex after TBI and UH. A, photomicrography of a coronal section at the bregma level from a sham-operated rat. B, photomicrography of a coronal section at the level $-4 \mathrm{~mm}$ from the bregma from a vehicle treated rat at $4 \mathrm{~h}$ after TBI and UH. Note that neuronal damage (swollen, shrunk, or dark eosin-stained, and so on) were present at $4 \mathrm{~h}$ after TBI + UH. C, photomicrography of a coronal section at the level $-4 \mathrm{~mm}$ from the bregma from a ghrelin $(4 \mathrm{nmol} / \mathrm{rat})$ treated rat at $4 \mathrm{~h}$ after TBI and UH. D, photomicrography of a coronal section at the level $-4 \mathrm{~mm}$ from the bregma from a ghrelin ( $8 \mathrm{nmol} / \mathrm{rat}$ )-treated rat at $4 \mathrm{~h}$ after TBI and UH. E, photomicrography of a coronal section at the level $-4 \mathrm{~mm}$ from the bregma from a ghrelin $(16 \mathrm{nmol} / \mathrm{rat})$-treated rat at $4 \mathrm{~h}$ after TBI and UH. F, percentage of damaged neurons in the cortex in sham-operated animals (Sham) and TBI $+\mathrm{UH}$ animals treated with normal saline (Vehicle) or ghrelin (Ghrelin) at $4 \mathrm{~h}$ after TBI $+\mathrm{UH}$. Data are presented as means \pm SE $(n=3)$ and compared with one-way ANOVA and StudentNewman-Keuls test: ${ }^{*} P<0.05$ versus Sham group; ${ }^{\#} P<0.05$ versus Vehicle group. Original magnification: 400x, Scale bar $=25 \mu \mathrm{m}$.

\section{Effects of Ghrelin on Morphological Alterations in the Brain after Traumatic Brain Injury and Uncontrolled Hemorrhagic Shock}

H-E staining showed neuronal damage (swollen, shrunk, or dark eosin-stained, and so on) at $4 \mathrm{~h}$ after TBI-UH in vehicletreated animals (Figure 3B) as compared with normal brain histology from a sham-operated animal (Figure 3A). All three doses of ghrelin treatment markedly reduced these damages at $4 \mathrm{~h}$ after TBI and UH (Figures 3C-E). As indicated in Figure 3F, the percentage of damaged neurons increased from $1.8 \pm 0.2 \%$ in sham-operated animals to $36.2 \pm 1.1 \%$ in vehicle treated animals at $4 \mathrm{~h}$ after TBI and UH $(P<0.05)$. Administration of ghrelin after TBI and $\mathrm{UH}$ reduced the percentage of damaged neurons in a dose-dependent manner (Figure 3F).

\section{Effects of Ghrelin on Axonal Damage after Traumatic Brain Injury and Uncontrolled Hemorrhagic Shock}

Immunohistochemistry with $\beta$-amyloid precursor protein ( $\beta$-APP), a marker of axonal damage (31), showed strong positive staining in vehicle treated rats at $4 \mathrm{~h}$ after TBI and UH (Figures 4B, F) as compared with that in sham-operated animals (Figures 4A, F). Ghrelin treatment reduced $\beta$-APP staining after TBI and UH in a dose-dependent manner (Figures 4C-F).

\section{Effects of Ghrelin on Sensorimotor and Reflex Function after Traumatic Brain Injury and Uncontrolled Hemorrhagic Shock}

As shown in Figure 5A, beam balance scores increased markedly in both vehicle- and ghrelin-treated animals at $1.5 \mathrm{~h}$ and $4 \mathrm{~h}$ after TBI and UH. Ghrelin-treated 


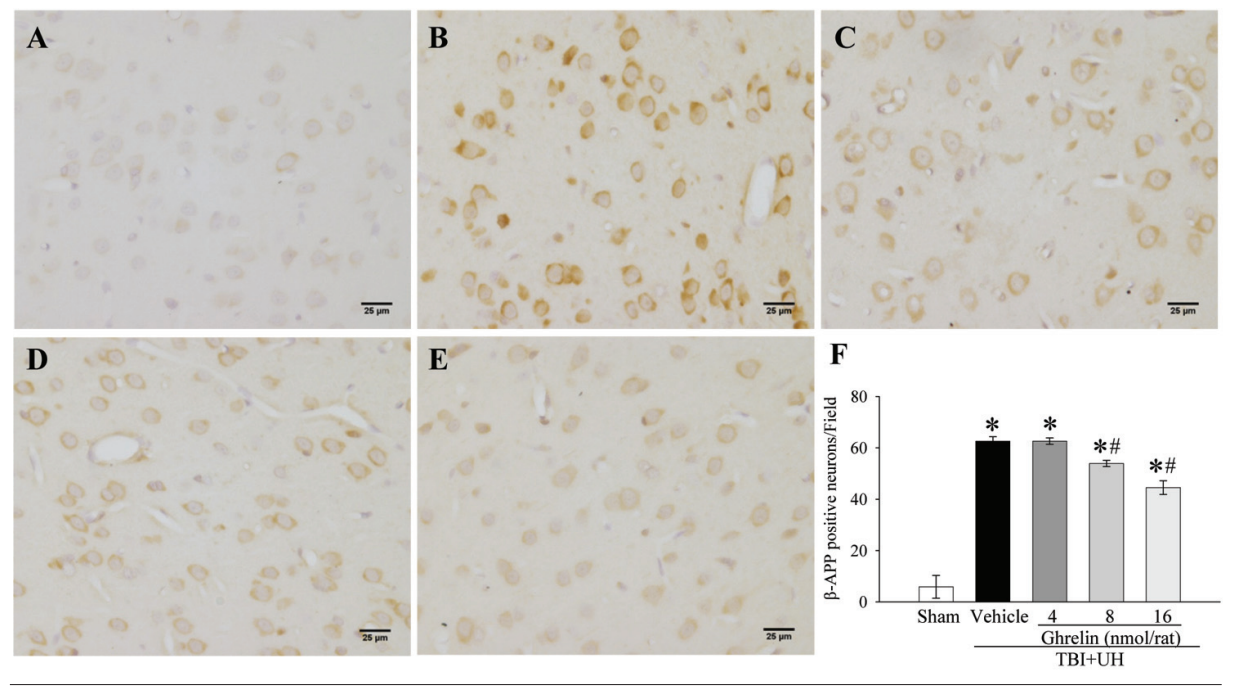

Figure 4. Effects of ghrelin on $\beta$-APP levels in the cortex after TBI and UH. A, $\beta$-APP immunohistochemical staining of a coronal section at the level $-4 \mathrm{~mm}$ from the bregma from a shamoperated rat. B, APP immunohistochemical staining of a coronal section at the level $-4 \mathrm{~mm}$ from the bregma from a vehicle treated rat at $4 \mathrm{~h}$ after TBI and UH. C, APP immunohistochemical staining of a coronal section at the level $-4 \mathrm{~mm}$ from the bregma from a ghrelin ( $4 \mathrm{nmol} / \mathrm{rat}$ )-treated rat at $4 \mathrm{~h}$ after TBI and UH. D, APP immunohistochemical staining of a coronal section at the level $-4 \mathrm{~mm}$ from the bregma from a ghrelin $(8 \mathrm{nmol} / \mathrm{rat})$-treated rat at $4 \mathrm{~h}$ after TBI and UH. E, APP immunohistochemical staining of a coronal section at the level $-4 \mathrm{~mm}$ from the bregma from a ghrelin (16 nmol/rat)-treated rat at $4 \mathrm{~h}$ after TBl and $\mathrm{UH}$. F, $\beta$-APP positive cell counts in sham-operated animals (Sham) and $\mathrm{TBI}+\mathrm{UH}$ animals treated with normal saline (Vehicle) or ghrelin (Ghrelin) at $4 \mathrm{~h}$ after TBI + UH. $\beta$-APP positive cells were counted at $200 \times$ original magnification. All the dark yellow or brown staining was counted as positive. However, some thin or faded yellow staining as shown in the sham operated animal was considered as background. The average number of positive cells in 4 random fields was determined. Data are presented as means $\pm \operatorname{SE}(n=3)$ and compared with one-way ANOVA and Student-Newman-Keuls test: ${ }^{*} P<0.05$ versus Sham group; $P<$ 0.05 versus Vehicle group. Original magnification: 400x, Scale bar $=25 \mu \mathrm{m}$.

animals had much lower beam balance scores than vehicle-treated animals. All three doses of ghrelin treatment significantly improved beam balance scores at both $1.5 \mathrm{~h}$ and $4 \mathrm{~h}$ after TBI and UH. For the forelimb placing test, slight improvements were found in the $4 \mathrm{nmol} / \mathrm{rat}$ ghrelin-treated animals at both 1.5 and $4 \mathrm{~h}$ after TBI and UH (Figure 5B). However, these improvements were not statistically significant at $1.5 \mathrm{~h}$ after TBI and UH. When 8 or $16 \mathrm{nmol} / \mathrm{rat}$ ghrelin was administered, all the improvements were statistically significant (Figure 5B). For the hindlimb placing test, on the other hand, only the $16 \mathrm{nmol} /$ rat ghrelin group showed a significant improvement at both 1.5 and $4 \mathrm{~h}$ after TBI and UH (Figure $5 \mathrm{C}$ ). To determine long-term effects of ghrelin on neurological damage following TBI and uncontrolled hemorrhage, ghrelin (16 nmol / rat) or vehicle (1 mL normal saline) was s.c. administered daily for $10 \mathrm{~d}$ starting at $4 \mathrm{~h}$ after TBI and UH. Neurological functional impairments and improvements were measured daily for up to $28 \mathrm{~d}$ after TBI and $\mathrm{UH}$ with or without ghrelin treatment. As shown in Figure 6A, although beam balvehicle- and ghrelin-treated animals, ghrelin-treated animals had much lower animals. Moreover, it took $19 \mathrm{~d}$ for beam balance scores to return to normal in the vehicle-treated animals. Beam balance scores in ghrelin-treated animals returned to normal in $11 \mathrm{~d}$. As shown in ance scores increased markedly in both beam balance scores than vehicle-treated

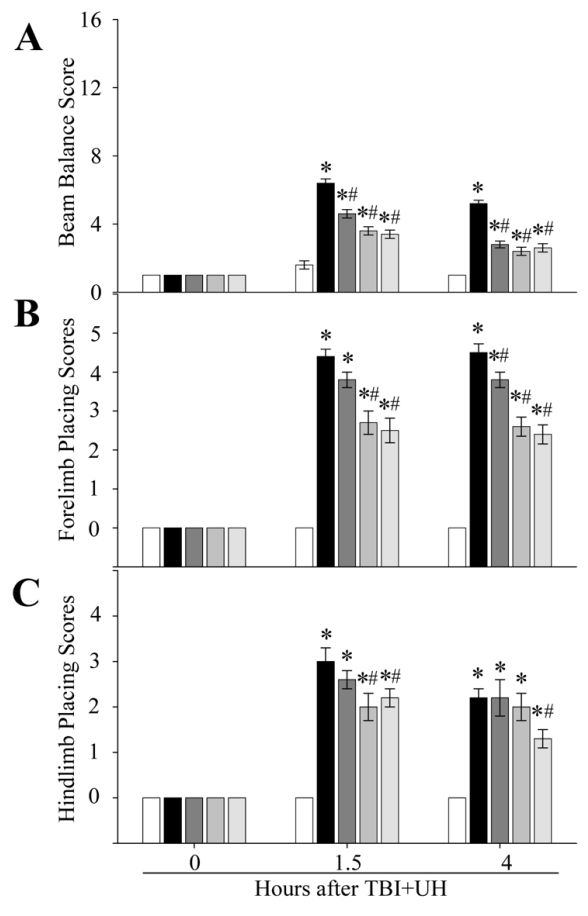

Figure 5. Improvement of short-term sensorimotor and reflex functions by ghrelin after TBI and UH. Alterations in beam balance scores (A), forelimb placing scores (B) and hindlimb placing scores (C) in sham-operated animals and $\mathrm{TBI}+\mathrm{UH}$ animals treated with normal saline (vehicle) or ghrelin at 1.5 and $4 \mathrm{~h}$ after TBI $+\mathrm{UH}$. Data are presented as means $\pm \operatorname{SE}(n=6)$ and compared with one-way ANOVA and Student-Newman-Keuls test: * $P<0.05$ versus sham group: ${ }^{~} P<0.05$ versus vehicle group. $\square$, Sham; $\mathbf{\square}, \mathrm{TBI}+\mathrm{UH}$ (vehicle); $\square, \mathrm{TBI}+\mathrm{UH}$ (ghrelin, $4 \mathrm{nmol} / \mathrm{rat}$ ); $\square, \mathrm{TBI}+\mathrm{UH}$ (ghrelin, $8 \mathrm{nmol} / \mathrm{rat}$ ); $\square, \mathrm{TBI}+\mathrm{UH}$ (ghrelin, $16 \mathrm{nmol} / \mathrm{rat})$.

Figure 6B, forelimb placing scores increased significantly after TBI and UH. Ghrelin treatment significantly reduced forelimb placing scores after TBI and UH as compared with vehicle treatment (Figure 6B). Similarly, hindlimb placing scores increased significantly after TBI and UH (Figure 6C). Ghrelin-treated animals had significantly lower hindlimb placing scores than vehicle treated animals. Hindlimb placing scores also returned to normal quicker in ghrelintreated animals than vehicle-treated animals (Figure 6C). 


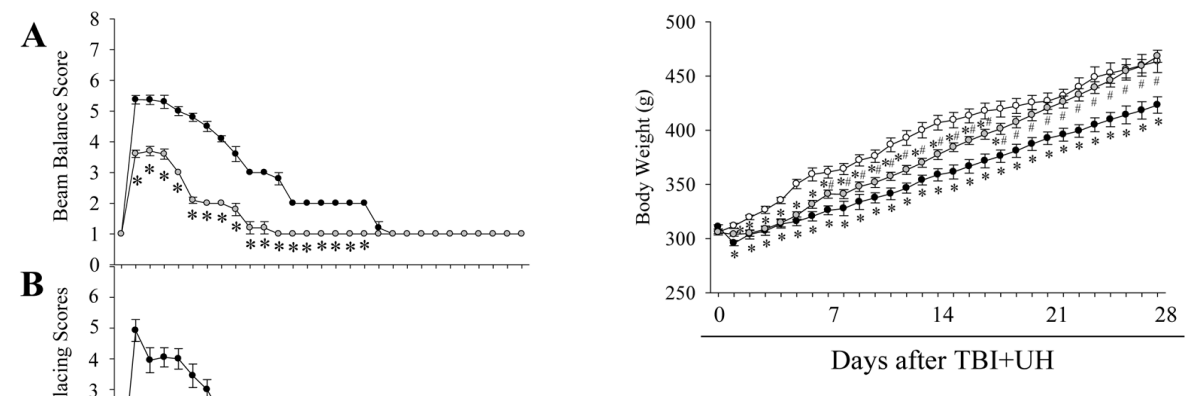

Figure 7. Increase in body weight by ghrelin after TBI and UH. Alterations in body weight in sham animals ( $-\bigcirc-, n=5), \mathrm{TBI}+$ $\mathrm{UH}$ animals treated with normal saline (- - - vehicle, $n=26$ ) or $16 \mathrm{nmol} / \mathrm{rat} /$ day ghrelin ( - -,$n=18$ ) for $10 \mathrm{~d}$. Data are presented as means \pm SE and compared by one-way ANOVA and Student-NewmanKeuls test: * $P<0.05$ versus sham group; ${ }^{\#} P<0.05$ versus vehicle group.

Days after TBI+UH

Figure 6. Improvement of long-term sensorimotor and reflex functions by ghrelin after TBI and UH. Alterations in beam balance scores (A), forelimb placing scores (B) and hindlimb placing scores (C) in $\mathrm{TBI}+\mathrm{UH}$ animals treated with normal saline (-—, vehicle, $n=26$ ) or $16 \mathrm{nmol} / \mathrm{rat} / \mathrm{day}$ ghrelin (—-,$n=18$ ) for $10 \mathrm{~d}$ after TBI + $\mathrm{UH}$. Data are presented as means $\pm \mathrm{SE}$ and compared by Student $t$ test: ${ }^{*} P<0.05$ versus vehicle group.

\section{Effects of Ghrelin on Body Weight Gain after Traumatic Brain Injury and Uncontrolled Hemorrhagic Shock}

As shown in Figure 7, sham-operated rats gained $5.6 \pm 0.60$ g every day. A significant drop in body weight was observed at $\mathrm{d} 1$ after TBI and UH in vehicle-treated animals. Then, the animals started to gain body weight at $\mathrm{d} 2$ after TBI and UH, however, at a slower pace ( $4.7 \pm 0.27$ g every day) than shamoperated animals. At $28 \mathrm{~d}$ after TBI and $\mathrm{UH}$, the body weight of vehicle treated animals was $8.7 \%$ lower than that of sham-operated animals. A slight drop in body weight was observed at $\mathrm{d} 1$ after TBI and UH in ghrelin-treated animals. Moreover, ghrelin-treated animals gained body weight at a quicker pace (6.1 \pm 0.42 g every day) than vehicle-treated animals after TBI and UH. At $d 7$ after TBI and $\mathrm{UH}$, the body weight in ghrelintreated animals were significantly higher than that in vehicle-treated animals. At d 28 after TBI and UH, the body weight in ghrelin-treated animals was even slightly higher than that of shamoperated animals.

\section{Effects of Ghrelin on Survival after Traumatic Brain Injury and Uncontrolled Hemorrhagic Shock}

As shown in Figure 8, the survival rate after TBI and UH was $73 \%$ at $\mathrm{d} 1$ and reduced to $66 \%$ at $\mathrm{d} 3-28$. On the other hand, there was no mortality in ghrelintreated TBI and UH animals during the $28 \mathrm{~d}$ observation period $(P<0.05)$. The sham-operated animals also had a $100 \%$ survival rate.

\section{DISCUSSION}

Novel therapeutic advances in the field of trauma management depend upon animal models that can accurately predict the clinical efficacy of interventions. In the battlefield, TBI injuries occur as a direct result of blast waves (primary blast injury), impact from objects put in motion by the blast (secondary blast injury), and by people being forcefully put in motion by the blast (tertiary blast injury). The brain is clearly vulnerable to

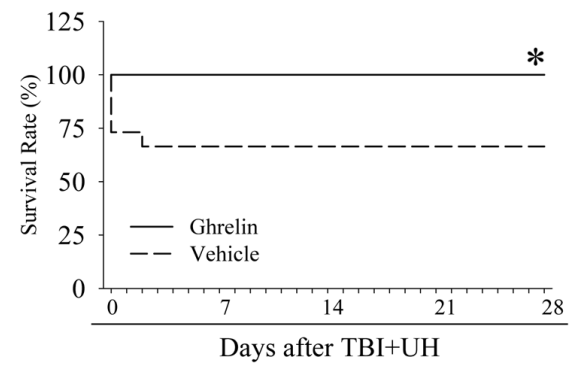

Figure 8. Reduction in mortality by ghrelin after TBI and UH. Alterations in survival rates in $\mathrm{TBI}+\mathrm{UH}$ animals treated with normal saline (Vehicle, $n=26$ ) or $16 \mathrm{nmol} / \mathrm{rat} /$ day ghrelin (Ghrelin, $n=18$ ) for $10 \mathrm{~d}$. The survival rate was estimated by KaplanMeier method and compared by the logrank test: ${ }^{*} P<0.05$ versus Vehicle group.

both secondary and tertiary blast injury. The most common type of TBI in the battlefield is diffuse axonal injury. In this regard, we chose to use Marmarou's acceleration impact model to induce head trauma in the present study. This model produces a pronounced diffuse cellular and axonal injury in forebrain structures such as sensorimotor cortex and hippocampus but limited brainstem damage $(23,24)$. Posttraumatic ventriculomegaly is observed in survivors at 4-6 wk after injury, which mirrors the experience in human head injury. In addition, TBI and hemorrhagic shock often occur concomitantly in the battlefield due to multiple injuries. In the current study, we determined the efficacy of ghrelin in a highly military relevant experimental rat model of TBI combined with uncontrolled hemorrhagic shock. To mimic the far-forward battlefield situation, the first dose of ghrelin was intravenously administered together with low volume crystalloid resuscitation (that is, field resuscitation) at 45 min after TBI and hemorrhage. To study the long-term effects of ghrelin after TBI and UH, ghrelin or vehicle was s.c. administered daily for $10 \mathrm{~d}$ after TBI and $\mathrm{UH}$ in additional groups of animals. The animals were observed for up to $28 \mathrm{~d}$ after TBI and UH. Our results demonstrate that administration of ghrelin attenuated brain injury, improved brain 
functions, and reduced mortality in a rat model of TBI and uncontrolled hemorrhagic shock.

Gastrointestinal dysfunction occurs frequently in patients with TBI (32). More than $50 \%$ of patients with severe head injuries develop gastrointestinal dysfunction as manifested by vomiting, abdominal distention, delayed gastric emptying, esophageal reflux and decreased intestinal peristalsis. Failure to maintain gastrointestinal function is a significant cause of posttrauma morbidity and mortality. However, little is known about whether gastrointestinal hormones are involved in modulating neuronal integrity and function after TBI. Ghrelin is a novel gastrointestinal hormone, cleaved from a precursor, preproghrelin (33). It is principally synthesized in endocrine cells of the stomach, termed X/A-like or ghrelin cells, and particularly found in the gastric fundus. About 2/3 to 3/4 of circulating ghrelin is of gastric origin. Lesser concentrations of ghrelin are found throughout the small intestine. The biological effects of ghrelin are mediated through the ghrelin receptor (that is, GHSR-1a), a 7 transmembrane domain $\mathrm{Gq}$ protein coupled receptor $(9,34)$. Ghrelin is the only identified endogenous ligand for this receptor. The wide distribution of ghrelin receptors suggests multiple paracrine, autocrine and endocrine roles of ghrelin (35-38). It has been linked to the regulation of pituitary hormone secretion, feeding, energy homeostasis, gastrointestinal function, and cardiovascular and immune system $(16,33,39)$. The results from the current study suggest that ghrelin, a gastrointestinal hormone, may be associated with neuronal damage and recovery after TBI. A recent study has shown that ghrelin levels decreased in a mouse model of TBI (40). In this regard, the decreased level of ghrelin may contribute to the secondary brain injury under such conditions. In our future studies, we will define the contribution of ghrelin downregulation on brain injury after TBI and UH.

The protective effects of ghrelin after brain injury appear to be related to its antiinflammatory $(16,33,39-41)$ and anti- apoptosis $(20,42)$ properties. TBI initiates a cascade of inflammatory processes including the release of proinflammatory mediators (43), which contributes to the acute pathologic processes and long-term neuronal damage following TBI (28). In this article, we found that brain levels of TNF- $\alpha$ and IL- 6 increased significantly at $4 \mathrm{~h}$ after TBI and UH and ghrelin treatment dose dependently decreased brain levels of TNF- $\alpha$ and IL- 6 under such conditions. Therefore, the beneficial effects of ghrelin after TBI and UH appears to be partially related to the downregulation of inflammatory mediators under such conditions. Apoptosis is thought to play an important role in both acute and chronic brain injury. Both animal and human studies have shown substantial evidence of neuronal apoptosis after TBI (44-47). Clearly, therapies that protect neurons from apoptosis are of great importance, because the loss of these cells contributes to the loss of neurological function and can greatly impact outcome. Ghrelin has been shown to inhibit apoptosis in neuronal cells during oxygen-glucose deprivation (19), and protect cortical neuron against focal ischemia/reperfusion in rats (20). In the current study, we found that ghrelin reduced cortical apoptosis at $4 \mathrm{~h}$ after TBI and UH in a dose-dependent manner. In this regard, the antiapoptosis property of ghrelin also contributes to its protection after TBI and hemorrhage.

Ghrelin is a hormone with multiple functions. Several other properties of ghrelin may indirectly contribute to its neuroprotective effects after TBI. Ghrelin stimulates growth hormone (GH) secretion. Chronic GH insufficiency is a common complication following TBI, and is associated with depression and diminished quality of life (48). Therefore, ghrelin may benefit TBI patients through a GH-dependent mechanism. Moreover, ghrelin has been reported to impact insulin secretion and glucose metabolism $(49,50)$. Patients with brain injury are particularly vulnerable to high blood glucose concentrations $(51,52)$. Both hypo- and hyperglycemia affect prognosis in braininjured patients, and there is an increas- ing belief that strict glucose control may benefit these patients (53). In this regard, the impact of ghrelin on GH, insulin and glucose concentrations in this model also warrants further investigation.

\section{CONCLUSION}

In summary, ghrelin treatment attenuated brain injury, facilitated function recovery, and improved survival in a rat model of TBI and uncontrolled hemorrhage. The protective effects of ghrelin after TBI and uncontrolled hemorrhage appear to be related to its antiinflammatory and antiapoptosis properties. For patients with severe TBI, death is a major outcome and improving survival is an important goal. With the majority of patients suffering from a moderate to mild TBI, long-term recovery of control over movements and ability to learn and remember new facts / tasks is a primary concern. Since administration of ghrelin not only reduced mortality but also facilitated function recovery, it has a great potential to be further developed as an effective resuscitation approach for the trauma victims with brain injury and severe blood loss.

\section{ACKNOWLEDGMENTS}

This study was supported by a DoD / DRMRP Grant DR080669 (R Wu) and an NIH grant R21 NS072608 (R Wu).

\section{DISCLOSURE}

The authors declare that they have no competing interests as defined by Molecular Medicine, or other interests that might be perceived to influence the results and discussion reported in this paper.

\section{REFERENCES}

1. Davis KL, Joshi AV, Tortella BJ, Candrilli SD. (2007) The direct economic burden of blunt and penetrating trauma in a managed care population. J. Trauma 62:622-9.

2. Boulanger L, et al. (2007) Excess mortality, length of stay, and costs associated with serious hemorrhage among trauma patients: findings from the National Trauma Data Bank. Am. Surg. 73:1269-74.

3. Coronado VG, et al. (2011) Surveillance for traumatic brain injury-related deaths-United States, 1997-2007. MMWR Surveill. Summ. 60:1-32.

4. Wu X, et al. (2008) Epidemiology of traumatic 
brain injury in eastern China, 2004: a prospective large case study. J. Trauma 64:1313-9.

5. Katsaragakis S, et al. (2010) Traumatic brain injury in Greece: report of a countrywide registry. Brain Inj. 24:871-6.

6. Manley G, et al. (2001) Hypotension, hypoxia, and head injury: frequency, duration, and consequences. Arch. Surg. 136:1118-23.

7. Dewitt DS, Prough DS. (2009) Blast-induced brain injury and posttraumatic hypotension and hypoxemia. J. Neurotrauma. 26:877-87.

8. Chesnut RM, et al. (1993) The role of secondary brain injury in determining outcome from severe head injury. J. Trauma 34:216-22.

9. Kojima M, Hosoda H, Date Y, Nakazato M, Matsuo H, Kangawa K. (1999) Ghrelin is a growth-hormone-releasing acylated peptide from stomach. Nature 402:656-60.

10. Arvat E, et al. (2000) Preliminary evidence that Ghrelin, the natural GH secretagogue (GHS)receptor ligand, strongly stimulates $\mathrm{GH}$ secretion in humans. J. Endocrinol. Invest. 23:493-5.

11. Date Y, et al. (2000) Central effects of a novel acylated peptide, ghrelin, on growth hormone release in rats. Biochem. Biophys. Res. Commun. 275:477-80.

12. Nass R, et al. (2000) Intracerebroventricular administration of the rat growth hormone $(\mathrm{GH})$ receptor antagonist G118R stimulates GH secretion: evidence for the existence of short loop negative feedback of GH. J. Neuroendocrinol. 12:1194-9.

13. Cowley MA, Grove KL. (2004) Ghrelin—satisfying a hunger for the mechanism. Endocrinology. 145:2604-6.

14. Andrews ZB, et al. (2008) UCP2 mediates ghrelin's action on NPY / AgRP neurons by lowering free radicals. Nature 454:846-51.

15. Wu R, et al. (2008) Orexigenic hormone ghrelin attenuates local and remote organ injury after intestinal ischemia-reperfusion. PLoS ONE. 3:e2026.

16. Wu R, et al. (2007) Ghrelin down-regulates proinflammatory cytokines in sepsis through activation of the vagus nerve. Ann. Surg. 245:480-6.

17. Wu R, et al. (2007) Ghrelin inhibits sympathetic nervous activity in sepsis. Am. J. Physiol. Endocrinol. Metab. 293:E1697-E1702.

18. Wu R, et al. (2007) Ghrelin attenuates sepsisinduced acute lung injury and mortality in rats. Am. J Respir. Crit. Care Med. 176:805-13.

19. Chung H, et al. (2007) Ghrelin inhibits apoptosis in hypothalamic neuronal cells during oxygenglucose deprivation. Endocrinol. 148:148-59.

20. Miao Y, Xia Q, Hou Z, Zheng Y, Pan H, Zhu S. (2007) Ghrelin protects cortical neuron against focal ischemia/reperfusion in rats. Biochem. Biophys. Res. Commun. 359:795-800.

21. Cheyuo C, Wu R, Zhou M, Jacob A, Coppa G, Wang P. (2011) Ghrelin suppresses inflammation and neuronal nitric oxide synthase in focal cerebral ischemia via the vagus nerve. Shock 35:258-65.

22. Bansal V, et al. (2010) The hormone ghrelin prevents traumatic brain injury induced intestinal dysfunction. J. Neurotrauma 27:2255-60.
23. Marmarou A, Foda MA, van den BW, Campbell J, Kita H, Demetriadou K. (1994) A new model of diffuse brain injury in rats. Part I: Pathophysiology and biomechanics. J. Neurosurg. 80:291-300.

24. Foda MA, Marmarou A. (1994) A new model of diffuse brain injury in rats. Part II: Morphological characterization. J. Neurosurg. 80:301-13.

25. Wu R, et al. (2007) Adrenomedullin and adrenomedullin binding protein-1 prevent metabolic acidosis after uncontrolled hemorrhage in rats. Crit. Care Med. 35:912-8.

26. Wu R, Dong W, Zhou M, Cui X, Hank SH, Wang P. (2005) Ghrelin improves tissue perfusion in severe sepsis via downregulation of endothelin-1. Cardiovasc. Res. 68:318-26.

27. Wu R, et al. (2009) Ghrelin hyporesponsiveness contributes to age-related hyperinflammation in septic shock. Ann. Surg. 250:126-33.

28. Ziebell JM, Morganti-Kossmann MC. (2010) Involvement of pro- and anti-inflammatory cytokines and chemokines in the pathophysiology of traumatic brain injury. Neurotherapeutics. 7:22-30.

29. Savitz SI, Rosenbaum DM. (1998) Apoptosis in neurological disease. Neurosurgery. 42:555-72.

30. Chaitanya GV, Steven AJ, Babu PP. (2010) PARP-1 cleavage fragments: signatures of celldeath proteases in neurodegeneration. Cell Commun. Signal. 8:31.

31. Medana IM, Esiri MM. (2003) Axonal damage: a key predictor of outcome in human CNS diseases. Brain 126:515-30.

32. Krakau K, Omne-Ponten M, Karlsson T, Borg J. (2006) Metabolism and nutrition in patients with moderate and severe traumatic brain injury: A systematic review. Brain Inj. 20:345-67.

33. Wang G, Lee HM, Englander E, Greeley GH, Jr. (2002) Ghrelin-not just another stomach hormone. Regul. Pept. 105:75-81.

34. Van Der Lely AJ, Tschop M, Heiman ML, Ghigo E. (2004) Biological, physiological, pathophysiological, and pharmacological aspects of ghrelin. Endocr. Rev. 25:426-57.

35. Hattori N, Saito T, Yagyu T, Jiang BH, Kitagawa K, Inagaki C. (2001) GH, GH receptor, GH secretagogue receptor, and ghrelin expression in human T cells, B cells, and neutrophils. J. Clin. Endocrinol. Metab. 86:4284-91.

36. Sakata I, Yamazaki M, Inoue K, Hayashi Y, Kangawa K, Sakai T. (2003) Growth hormone secretagogue receptor expression in the cells of the stomach-projected afferent nerve in the rat nodose ganglion. Neurosci. Lett. 342:183-6.

37. Papotti M, et al. (2000) Growth hormone secretagogue binding sites in peripheral human tissues. J. Clin. Endocrinol. Metab. 85:3803-7.

38. Shuto Y, et al. (2001) Generation of polyclonal antiserum against the growth hormone secretagogue receptor (GHS-R): evidence that the GHS-R exists in the hypothalamus, pituitary and stomach of rats. Life Sci. 68:991-6.

39. Kojima M, Kangawa K. (2005) Ghrelin: structure and function. Physiol. Rev. 85:495-522.

40. Bansal V, et al. (2012) Vagal stimulation modulates inflammation through a ghrelin mediated mechanism in traumatic brain injury. Inflammation. 35:214-20.

41. Wu R, et al. (2009) Ghrelin hyporesponsiveness contributes to age-related hyperinflammation in septic shock. Ann. Surg. 250:126-33.

42. Ammori JB, Zhang WZ, Li JY, Chai BX, Mulholland MW. (2008) Effects of ghrelin on neuronal survival in cells derived from dorsal motor nucleus of the vagus. Surgery 144:159-67.

43. Cederberg D, Siesjo P. (2010) What has inflammation to do with traumatic brain injury? Childs Nerv. Syst. 26:221-6.

44. Wong J, Hoe NW, Zhiwei F, Ng I. (2005) Apoptosis and traumatic brain injury. Neurocrit. Care 3:177-82

45. Luo C, Jiang J, Lu Y, Zhu C. (2002) Spatial and temporal profile of apoptosis following lateral fluid percussion brain injury. Chin. J. Traumatol. 5:24-7.

46. Clark RS, et al. (1999) Increases in Bcl-2 and cleavage of caspase- 1 and caspase- 3 in human brain after head injury. FASEB J. 13:813-21.

47. Dressler J, Hanisch U, Kuhlisch E, Geiger KD. (2007) Neuronal and glial apoptosis in human traumatic brain injury. Int. J. Legal Med. 121:365-75.

48. Kelly DF, et al. (2006) Neurobehavioral and quality of life changes associated with growth hormone insufficiency after complicated mild, moderate, or severe traumatic brain injury. J. Neurotrauma 23:928-42.

49. Sangiao-Alvarellos S, Cordido F. (2010) Effect of ghrelin on glucose-insulin homeostasis: therapeutic implications. Int. J. Pept. 2010:234709.

50. Dezaki K, Sone H, Yada T. (2008) Ghrelin is a physiological regulator of insulin release in pancreatic islets and glucose homeostasis. Pharmacol. Ther. 118:239-49.

51. Bilotta F, Giovannini F, Caramia R, Rosa G (2009) Glycemia management in neurocritical care patients: a review. J. Neurosurg. Anesthesiol. 21:2-9.

52. Jacka MJ, Torok-Both CJ, Bagshaw SM. (2009) Blood glucose control among critically ill patients with brain injury. Can. J. Neurol. Sci. 36:436-42.

53. Godoy DA, Di NM, Rabinstein AA. (2010) Treating hyperglycemia in neurocritical patients: benefits and perils. Neurocrit. Care 13:425-38 